\title{
MICROTENSILE BOND STRENGTH OF GLASS IONOMER CEMENT TO SILVER FLUORIDE AND POTASSIUM IODIDE-TREATED CARIOUS PRIMARY DENTIN
}

\author{
Rania Abdallah Nasr* and Hany Mohamed Saber*
}

\begin{abstract}
Purpose: A case control study was performed to investigate the effect of silver diamine fluoride (SDF) and potassium iodide (KI) [Riva Star, SDI] on the microtensile bond strength between glass ionomer cement and carious primary dentin and to evaluate the mode of restoration failure.

Methods: Forty carious primary molars were sectioned into halves through the carious lesions and randomly allocated to test and control groups. The test specimens were treated with SDF and $\mathrm{KI}$ with concentration of $0.06 \mathrm{gm} / \mathrm{ml}$ fluoride ions and the control specimens with deionized water. The samples were stored in artificial saliva for 14 days in normal room temprature, and the dentin surfaces were conditioned and restored with Fuji IX GP Extra. After 24 hours in artificial saliva, the specimens were prepared for microtensile bond strength testing and stressed with tension at $1 \mathrm{~mm} /$ minute until failure. Mean bond strengths were compared using the paired test and the failure mode was recorded.
\end{abstract}

Results: The mean microtensile strength for the test group was $7.4 \mathrm{MPa}(5.1 \pm \mathrm{SD})$ and 6.3 $( \pm 4.6)$ for the control group ( $>>0.05)$. Most common failure mode was the mixed failure in both groups.

Conclusion: Silver diamine fluoride and potassium iodide does not adversely affect the bond strength between glass ionomer cement and carious primary dentin in vitro.

\section{INTRODUCTION}

Dental caries is one of the most common oral diseases of childhood and is considered as a major public health problem. Despite efforts made in every country to eliminate dental caries as much as possible, yet it is still considered as the most prevalent disease all over the world and the major cause behind tooth loss ${ }^{(1)}$.

Dental caries is defined as the localized damage of vulnerable dental hard tissues due to the acidic products produced from bacterial fermentation of dietary carbohydrates. The disease process

* Assistant Professor in Pediatric Dentistry and Dental Public Health Department, Faculty of Dentistry, Cairo University, Egypt. 
is initiated within the bacterial biofilm (dental plaque) that covers a tooth surface and then continues to spread toward the inner dental tissues. Unfortunately, the very early changes starting in the enamel often go undetectable both clinically and Radiographically ${ }^{(2)}$.

Dental caries is mainly caused due to an ecological imbalance in the physiological equilibrium between tooth minerals and oral microbial biofilms, but there are mainly four major factors that contribute in the development of caries, which are the presence of bacteria, the substrate available for the bacteria to ferment, the host's oral environment and the time required for such process.

The mechanism of the caries process involves the presence of endogenous bacteria (mainly mutans Streptococci and Lactobacillus species) in the biofilm which produce weak organic acids as a by-product of metabolism of fermentable carbohydrates ${ }^{(3)}$.

Since dental caries is considered one of the most prevalent diseases worldwide, it is important to know its risk factors. Risk factors for dental caries can be biological or behavioral factors or both. Biological or physical factors such as inadequate salivary flow and composition, high numbers of cariogenic bacteria, insufficient fluoride exposure, gingival recession, immunological components, need for special health care and genetic factors ${ }^{(4)}$. Behavioral factors include poor oral hygiene; poor dietary habits, frequent consumption of refined carbohydrates; frequent use of oral medications that contain sugar; and inappropriate methods of feeding infants ${ }^{(5)}$.

Early Childhood Caries (ECC) is one of the most common diseases among children and known to have different names and terms which are often used interchangeably such as early childhood tooth decay, early childhood caries, baby bottle-fed tooth decay, early childhood dental decay, comforter caries, nursing caries, maxillary anterior caries, rampant caries, and many more ${ }^{(6)}$.
Prevalence of ECC depends on many factors such as race, culture, socioeconomic status, life style, dietary pattern, and oral hygiene practices and also varies from country to country and from area to area. The incidence of ECC among children with deciduous teeth is 1.76 billion (95\% CI: 1.26 billion; 2.39 billion) ${ }^{(7)}$. A review of literature made stated that the prevalence of ECC is between 1 and $14 \%$ in most developed countries ${ }^{(8)}$. In addition, the prevalence of ECC in the USA was found to be between 3 and $6 \%$, which is consistent with the prevalence in other western countries ${ }^{(9)}$. However, the prevalence was found to be as high as $70 \%$ in less developed countries especially in low socioeconomic groups ${ }^{(10)}$. Furthermore, in studies made in some Middle Eastern countries, prevalence was found to be as high as $76 \%$ in Palestine and $83 \%$ in the United Arab of Emirates ${ }^{(11,12)}$.

Abbass et al. (2019) conducted a study in Egypt, where a total number of 369 Egyptian children and adolescents (age ranges from 3-18 years) were examined to assess the prevalence of dental caries, dental status was analyzed using decayed, missing and filled tooth index (dmft) for deciduous dentition and (DMFT) index for permanent dentition. For mixed dentition (deft) index was used, $d$ (decayed primary tooth indicated for filling), e (decayed primary tooth indicated for extraction) and $\mathrm{f}$ (filled primary tooth). The study revealed that $74 \%$ of the children had dental caries with mean dmft: $3.23 \pm 4.07$; deft: $4.21 \pm 3.21$; DMFT: $1.04 \pm 1.56^{(13)}$.

\section{Consequences of untreated ECC}

Unfortunately, ECC does not only affect child's teeth and cause pain but it also affects speech and eating, lowers the child's self-esteem due to bad appearance and affects the quality of life of children and their caregivers including financial and health implications ${ }^{(14)}$.

In a study made on 77 children in Brazil who had severe caries, it was found that, $72.7 \%$ had toothache, $49.4 \%$ had difficulty in eating and $26.0 \%$ missed school because of their teeth ${ }^{(15)}$. Another 
study conducted in Canada on 266 children, categorized into 2 groups, where 144 had ECC and 122 were caries-free, it was found that, children with ECC had lower haemoglobin levels and more odds for iron deficiency anaemia than caries free children ${ }^{(16)}$.

Moreover, the longer the ECC is left untreated, the more difficult it becomes to treat the child, the more the cost increases and the harder it becomes for the number of clinicians who can perform complicated procedure ${ }^{(2)}$. Tooth extraction hence can become an unavoidable necessary treatment option for ECC, resulting in early loss of molars, subsequently leading to future orthodontic problems, which increases the financial burdens on the child's parents.

Early childhood caries (ECC) should be considered as one example of the challenges. Behavioral issues routinely complicate or prevent restorative treatment of ECC in young children. Yet, if left untreated, the disease progresses producing pain, has a negative impact on the quality of life, and in extreme cases can be life threatening. Further, because of the barriers to accessing dental care, vulnerable populations go through life with untreated disease ${ }^{(17)}$.

Finally, Hakan et al. (2013) categorized all the possible consequences of ECC into 3 categories, short term, long term and rare sequelae. The short term sequelae include pain, infection, poor appetite, disturbed sleep, loss of school days with reduced ability to learn, possibly hospitalization and need for treatment under general anesthesia $(\mathrm{GA})^{(2)}$.

The long term consequences include poor oral health that continues to adulthood, probability to affect speech, nutrition, and the quality of life, higher risk of caries in succeeding permanent teeth and increased treatment costs and time for the parents. The rare sequelae include suborbital cellulites, brain abscesses and unexplained recurrent fevers ${ }^{(2)}$.
Systematic reviews of human randomized controlled trials now suggest multiple preventive interventions as alternates to the traditional methods of restorative care for ECC ${ }^{(17-19)}$. One of those interventions is Silver Diamine Fluoride, SDF, which is unique in both killing the bacteria and hardening the teeth, thus both arresting and preventing caries. It appears to be almost twice as effective as fluoride varnish for caries arrest ${ }^{(18)}$.

Studies have shown that silver diamine fluoride (SDF) has an intense antibacterial effect on cariogenic bacteria and can inhibit the growth of multi-species cariogenic biofilms on tooth surfaces (20-22). SDF is a topical fluoride which is often used in high concentration (38\%) for preventing and arresting dental caries ${ }^{(23)}$. SDF has recently been approved for clinical use by the United States Food and Drug Administration in 2015.

A review concluded SDF as an effective, efficient, equitable and safe caries-preventive agent appearing to meet the World Health Organization's Millennium Goals for 21st century medical care ${ }^{(18)}$. Clinical studies also showed the success of SDF in preventing and arresting dental caries ${ }^{(24,25)}$. A laboratory study found that the bond strength of restorations to dentine was not adversely affected by SDF using resin-based adhesives (26). The application of SDF under GIC restorations has been demonstrated to produce a promising pulpal response and be effective in facilitating the formation of reparative dentine ${ }^{(27)}$.

It also has been reported that prior treatment with SDF can increase resistance of cavity margins around GIC restorations to secondary caries development ${ }^{(28)}$.

A significant disadvantage of SDF use, however, is black staining on teeth which can cause aesthetic concern (24). A way that has been suggested of managing this problem is to apply a saturated solution of potassium iodide (KI) immediately after SDF application. It was suggested that discoloration 
of the carious lesion can be avoided while the caries arresting effect of SDF is not changed (29). The suggested explanation is that the silver ions from the SDF solution will react with the iodide ions from the KI solution to form silver iodide. It was reported that the application of SDF + KI to dentine surfaces before the placement of GIC restorations did not affect the bond strength of GIC to dentine ${ }^{(30)}$, and did not adversely interfere with the fluoride uptake into the adjacent demineralized dentine ${ }^{(29)}$.

It would be desirable if KI could inhibit the staining formation associated with SDF without diminishing its effectiveness in preventing and arresting dental caries. Nevertheless, evidence from laboratory data is still insufficient to support this claim.

Furthermore, the restorative material makes a huge difference in the clinical success of a restoration. Packable glass ionomer cement (GIC) due to its superior biocompatibility, chemical adhesion, anticariogenic property, resistance to wear and lower moisture sensitivity has been advocated to be used as restorations in deep caries management. GIC is also the material of choice in pediatric patients with active caries and high caries risk. ${ }^{(31)}$

Therefore, the purpose of this study was to investigate the effect of Silver Diamine Fluoride (SDF) with Potassium Iodide (KI) [Riva Star, SDI] on the microtensile bond strength between glass ionomer cement and treated carious primary dentin and to evaluate the mode of restoration failure.

\section{METHODS}

1. Forty carious primary teeth, extracted either due to shedding or due to orthodontic purposes, stored in a solution of 0.9 percent sodium chloride at room temperature for a maximum of two months, were used.

The teeth were obtained from the Outpatient Clinics of Pediatric Dentistry Department, Cairo
University, using a protocol that approved by the Ethics Committee of the Faculty of Dentistry, Cairo University, Egypt.

2. The primary teeth only with dentin caries that radiographically extended more than half the distance between the dentinoenamel junction and pulp chamber and with at least two surfaces of tooth structure remaining were selected. Figure (1)

3. Each tooth was sectioned through the center of the carious lesion using a slow-speed cutting machine (Isomet 1000, USA) forming two similar halves. Figure (2)

4. Following sectioning, these two specimens were randomly allocated to either the test or the control group.

5. In the test group, the carious dentin surfaces were treated with 38 percent SDF solution (Riva Star, SDI, Australia) using a micro-brush provided with the kit, followed by application of Potassium Iodide (KI) for 3 minutes till forming a creamy mix, followed by a 30 -second rinse with distilled water. Figure (3)

6. In the control group, deionized water was applied for three minutes. Figure (4)

7. All specimens were stored in artificial saliva for 14 days at $24^{\circ} \mathrm{C}$

8. After storage, the carious proximal surface of each specimen (test \& control) was reduced occluso-gingivally with a diamond bur in a high-speed hand-piece then polished and smoothed with a silicon carbide paper cup on a water-cooled Lathe to create a flat surface.

9. The SDF-treated samples were reduced, leaving a blackened dentin layer remaining to make sure a layer of SDF treated dentin is still remaining after flattening the surface.

10. The roots were removed from the remaining crown with a high-speed diamond bur under water-cooling. 


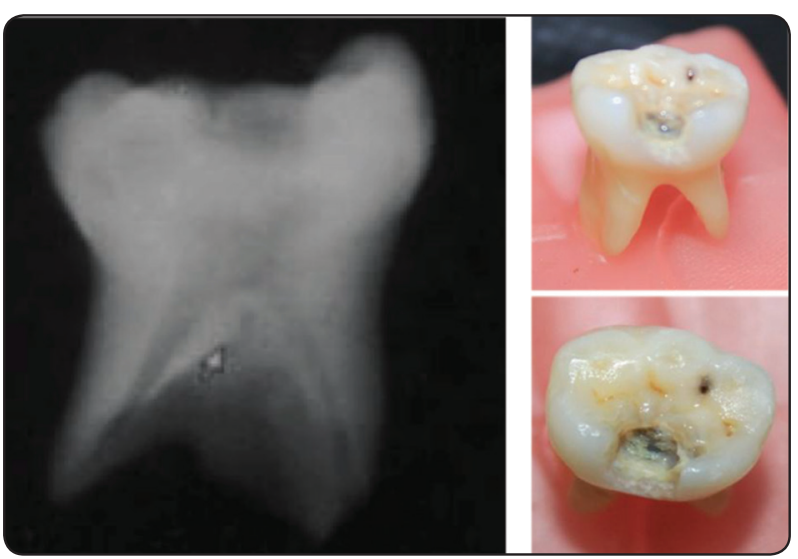

Fig. (1) Radiographically, Dentin caries extended more than half the distance between the dentinoenamel junction and pulp chamber and with at least two surfaces of tooth structure remaining.

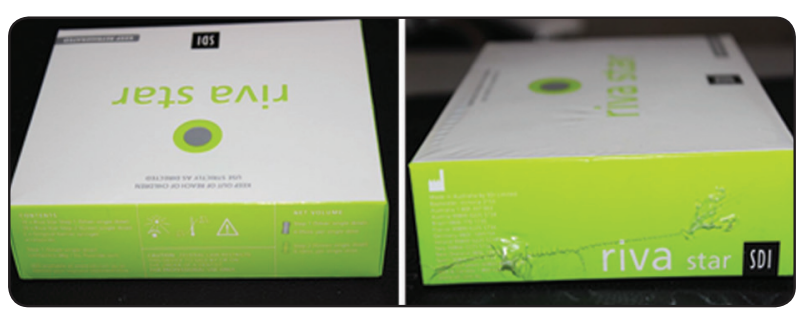

Fig. (3) SDF solution, 38\%, (Riva Star, SDI, Australia) with Potassium Iodide (KI) used with test specimen

11. An approximately four mm high GIC block (Fuji IX GP Extra Capsule, GC, Japan ) was built up on the dentin surface with a clear tape wrapped around the specimen as a temporary mold after being mixed via Amalgamator (Foshan, China) according to the manufacturer's instructions. Figure (5)

12. After the glass ionomer hardened (five minutes), the specimens were stored in artificial saliva at 37 degrees Celsius for 24 hours.

13. The specimens were then sectioned using a slow-speed water-cooled diamond saw (Isomet 1000 , USA) to obtain slices approximately 0.7 $\mathrm{mm}$ thick, which were fixed in Acrylic blocks.

Figure (6) a

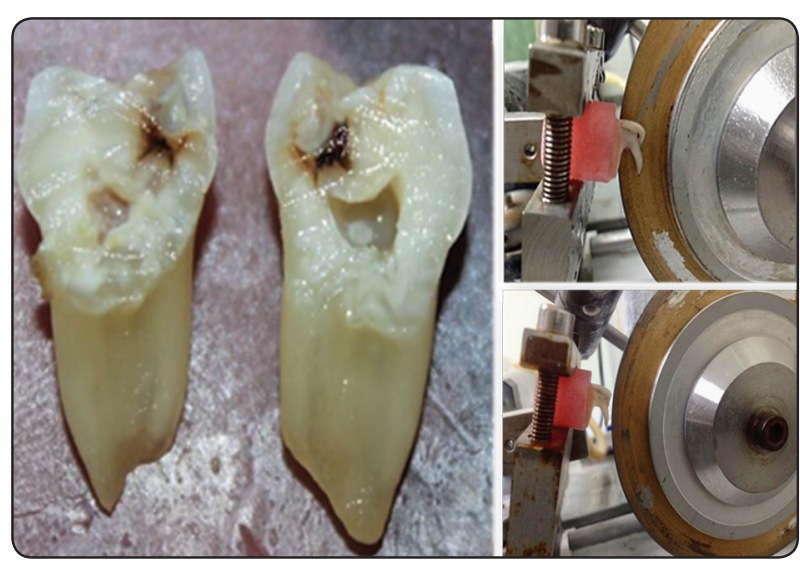

Fig. (2) Each tooth was sectioned through the center of the carious lesion using a slow-speed cutting machine (Isomet 1000, USA) forming two similar halves.

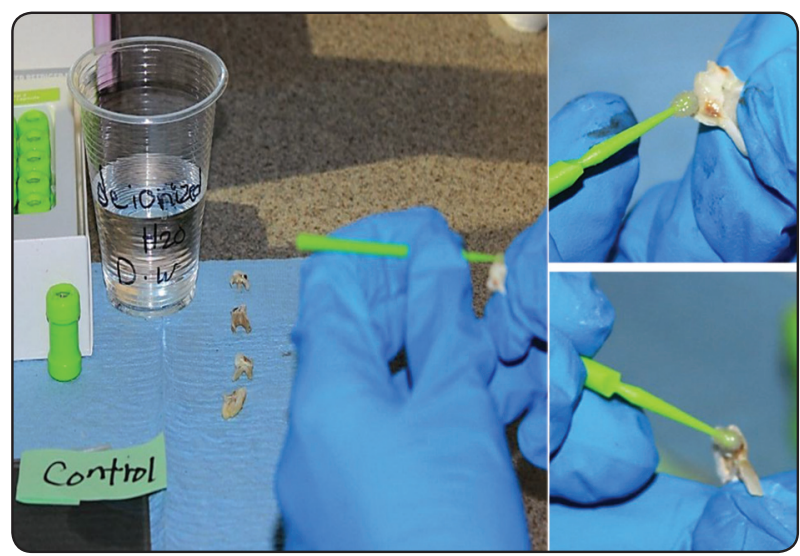

Fig. (4) In the test group, the carious dentin surfaces were treated with 38 percent SDF solution, followed by application of Potassium Iodide (KI). In the control group, deionized water was applied for three minutes.

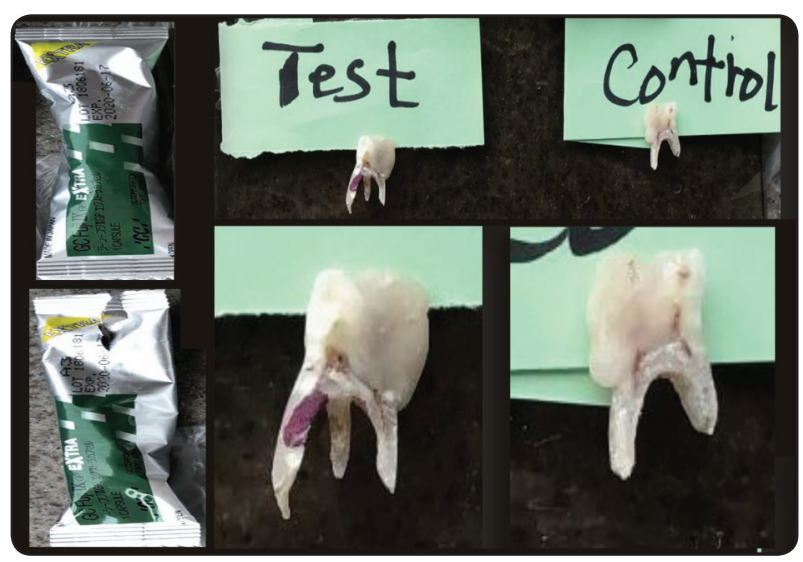

Fig. (5) About four mm high GIC block (Fuji IX GP Extra Capsule, GC, Japan) was built up on the dentin surface. 
14. The specimens were trimmed and prepared into an hour-glass shape with a cross-sectional area of one $\mathrm{mm} 2$ using a tapered diamond bur Figure (6) b. The SDF treated specimens were prepared such that only the blackened dentin/ GIC interface was at middle of the hour-glass shape. Figure (6) c.

15. Specimens were kept wet during the shaping and testing procedures.

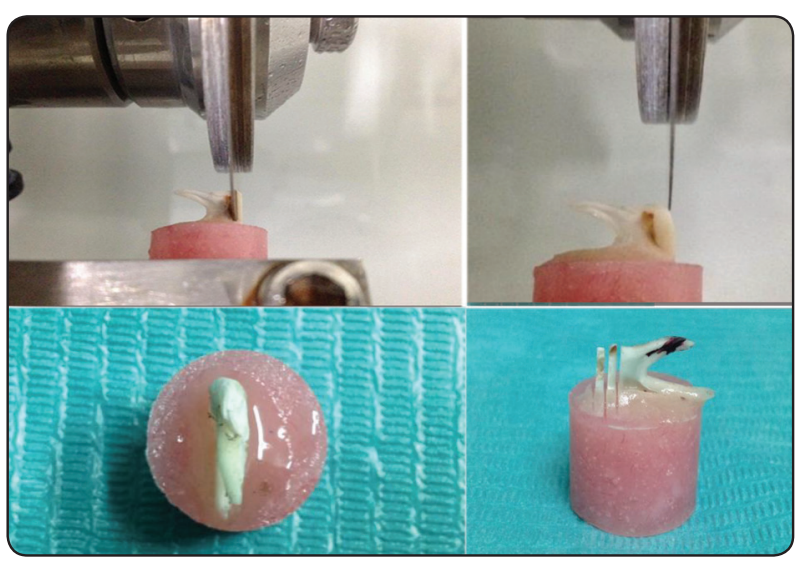

Fig. (6a) The specimens were trimmed and prepared into an hour-glass shape with a cross-sectional area of one $\mathrm{mm} 2$ using a tapered diamond bur
16. Each specimen was placed in the testing jig of a Universal Testing Machine (Shimadzu model EZ-S500, Kyoto, Japan) and stressed in tension at a crosshead speed of one mm per minute until bond failure. Figure (7)

The maximum stress at failure was recorded and converted to mega Pascal units (MPa). Force gauge readings in $\mathrm{kgf}$ were converted to MPa using the following equation: $1 \mathrm{kgf} / \mathrm{mm} 2=$ 9.80665 MPa.

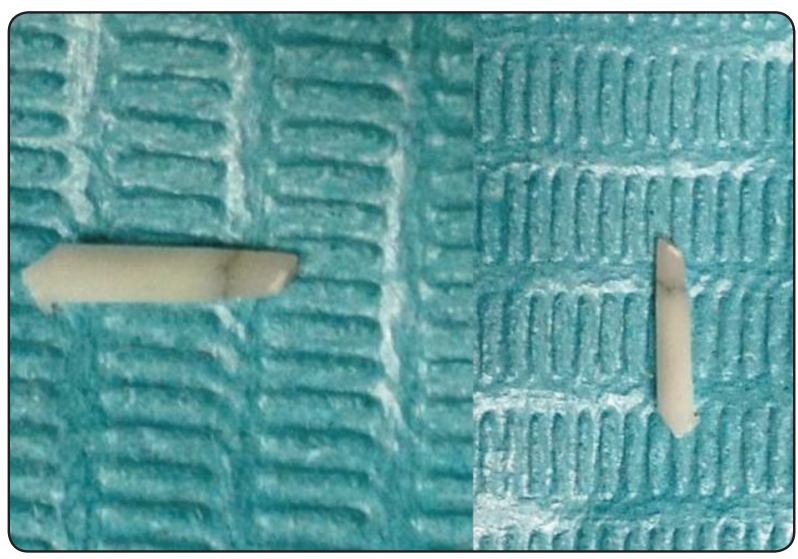

Fig. $(6$ b,c) The specimens were then sectioned using a slowspeed water-cooled diamond saw (Isomet 1000, USA) to obtain slices approximately $0.7 \mathrm{~mm}$ thick, which were fixed in Acrylic blocks.

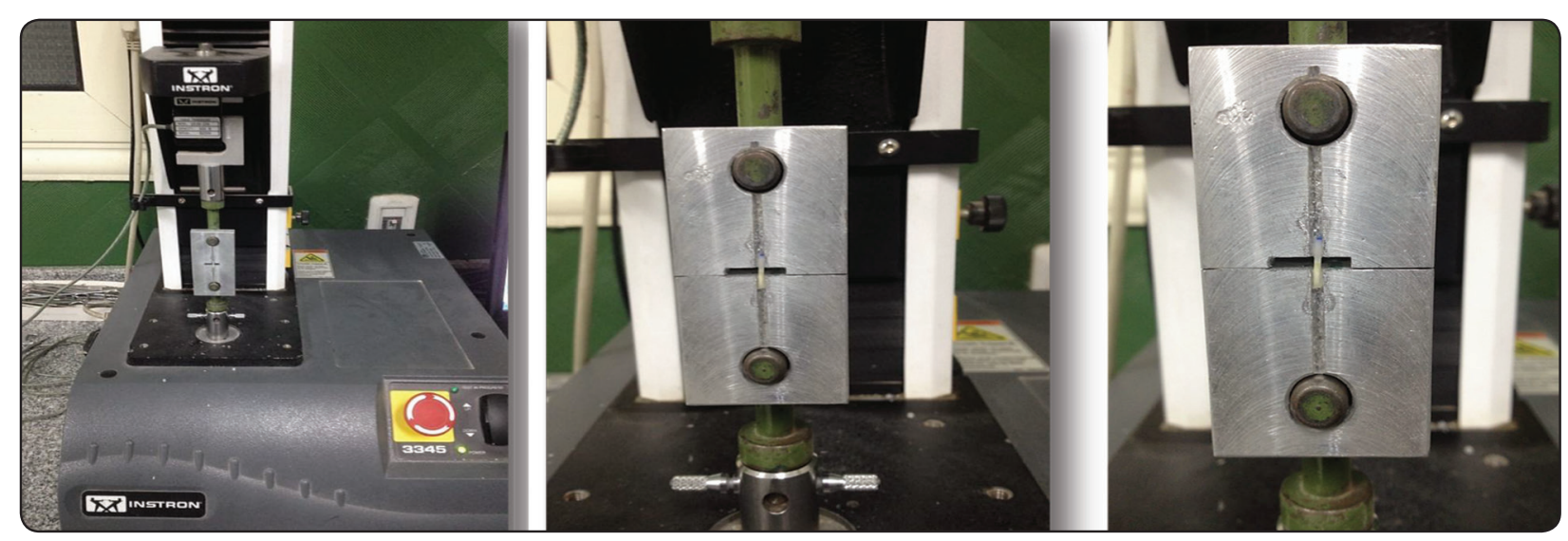

Fig. (7) Universal Testing Machine(Shimadzu model EZ-S500, Kyoto, Japan) and each specimen is stressed in tension at a crosshead speed of one mm per minute until bond failure. 
17. According to the method for microtensile bond strength testing, one beam derived from each tooth specimen was used as the value representing for that specimen.

18. The bond strength for each tooth was being determined by the bond strength of the beam derived from each tooth.

19. The failure mode was observed and recorded with a magnifying microscope (MEIJI ML 9300, Saitama, Japan), and the modes were classified into four groups:

(1) Adhesive failure between the restorative material and dentin surface;

(2) Cohesive failure in dentin;

(3) Cohesive failure in the restorative material; and

(4) Mixed failure, which was a combination of adhesive failure (between the adhesive and dentin) and cohesive failure (within the restorative material).

\section{Statistical Analysis:}

Categorical data was presented as frequencies and percentages and was analyzed using chi square test. Numerical data was explored for normality by checking the data distribution, calculating the mean and median values and using Kolmogorov-Smirnov and Shapiro-Wilk tests. Data showed parametric distribution so; it was presented by mean and standard deviation (SD) values and was analyzed using paired t-test. The significance level was set at $\mathrm{P} \leq 0.05$ within all tests. Statistical analysis was performed with IBM $^{\circledR}$ SPSS $^{\circledast}$ Statistics Software Version 25 for Windows. (IBM Corp., Armonk, N.Y., USA).

\section{RESULTS}

\section{I-Micro-tensile bond strength:}

\section{Descriptive Statistics:}

Descriptive statistics for both groups were presented in Table (1) and Figure (8).

TABLE (1): Descriptive statistics for micro-tensile bond strength (Mpa) for both groups

\begin{tabular}{|c|c|c|c|c|}
\hline Material & Mean & $\begin{array}{c}\text { Std. } \\
\text { Deviation }\end{array}$ & Median & Range \\
\hline Control & 7.94 & 1.25 & 7.84 & 6.83 \\
\hline Test & 8.31 & 2.49 & 8.16 & 9.88 \\
\hline
\end{tabular}

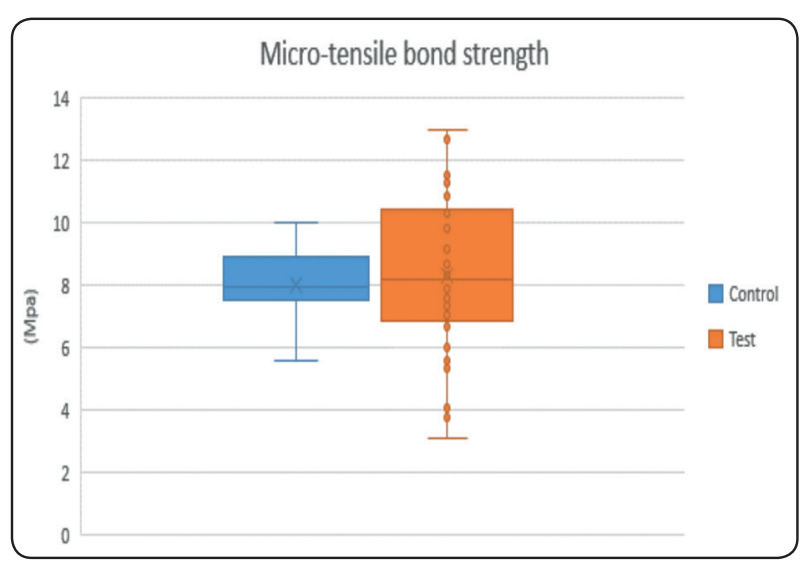

Fig. (8): Box plot showing micro-tensile bond strength (Mpa) for both groups

\section{Intergroup Comparison:}

Paired t-test showed that test samples $(8.31 \pm 2.49)$ had a non-significant higher micro-tensile bond strength than control samples $(7.94 \pm 1.25)$ $(\mathrm{P}=0.479)$. Mean and standard deviation (SD) values of micro-tensile bond strength (Mpa) for both groups were presented in Table (2) and Figure (9).

\footnotetext{
${ }^{\circledR}$ IBM Corporation, NY, USA.

${ }^{\circledR}$ SPSS, Inc., an IBM Company.
} 
TABLE (2): Mean \pm standard deviation (SD) of micro-tensile bond strength (Mpa) for both groups

\begin{tabular}{|c|c|c|c|c|c|}
\hline \multicolumn{2}{|c|}{ Material (mean $\pm S D)$} & \multirow{2}{*}{$\begin{array}{c}\text { Mean } \\
\text { difference }\end{array}$} & \multirow{2}{*}{$D f$} & \multirow{2}{*}{ t-value } & \multirow{2}{*}{$P$-value } \\
\hline Control & Test & & & & \\
\hline $7.94 \pm 1.25$ & $8.31 \pm 2.49$ & -0.37 & 39 & -0.714 & $0.479 \mathrm{~ns}$ \\
\hline
\end{tabular}

$D f$ : Degree of freedom $*$; significant $(p \leq 0.05)$

$n s ;$ non-significant ( $p>0.05)$

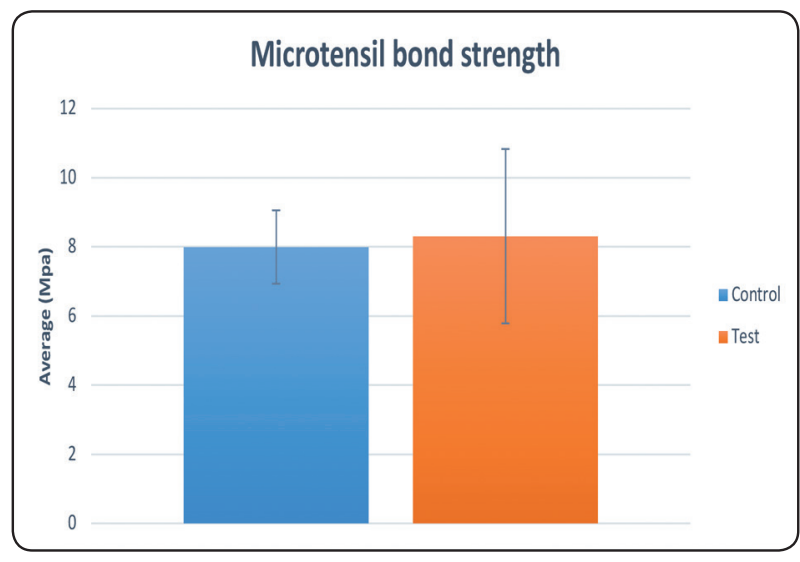

Fig, (9): Bar chart showing average micro-tensile bond strength (Mpa) for both groups

\section{II-Mode of failure}

\section{Mode of failure in both groups:}

Chi square test showed that there was no significant difference in the distribution of the mode of failure in both groups $(\mathrm{P}=0.301)$. Majority of the control samples (50.00\%) (20) had a mixed failure, $(17.50 \%)$ (7) failed adhesively and (17.50\%) (7) failed cohesively in glass ionomer, while only (15.00\%) (6)

failed cohesively in dentin. Most of the test samples (45.00\%) (18) had a mixed failure, (35.00\%) (14) failed adhesively, while only (10.00\%) (4) of the samples had a cohesive failure in dentine and glass ionomer. Frequencies (n) and percentages (\%) for mode of failure in both groups were presented in Table (3) and Figures from (10 to 12).

TABLE (3): Frequencies (n) and Percentages (\%) of mode of failure in both groups

\begin{tabular}{|c|c|c|c|c|c|c|c|}
\hline \multirow{2}{*}{ Failure mode } & \multicolumn{2}{|c|}{ Control } & \multicolumn{2}{c|}{ Test } & \multicolumn{2}{c|}{ Total } & P-value \\
\cline { 2 - 7 } & $\%$ & $\mathrm{n}$ & $\%$ & $\mathrm{n}$ & $\%$ & $\mathrm{n}$ \\
\hline Mixed & $50.00 \%$ & 20 & $45.00 \%$ & 18 & $47.50 \%$ & 38 \\
\hline Adhesive & $17.50 \%$ & 7 & $35.00 \%$ & 14 & $26.25 \%$ & 21 \\
\hline Cohesive in dentine & $15.00 \%$ & 6 & $10.00 \%$ & 4 & $12.50 \%$ & 10 \\
\hline Cohesive in GI & $17.50 \%$ & 7 & $10.00 \%$ & 4 & $13.75 \%$ & 11 \\
\hline Total & $100 \%$ & 40 & $100 \%$ & 40 & $100 \%$ & 80 \\
\hline
\end{tabular}

*; Significant $(p \leq 0.05) n s ;$ Non-Significant $(p>0.05)$ 


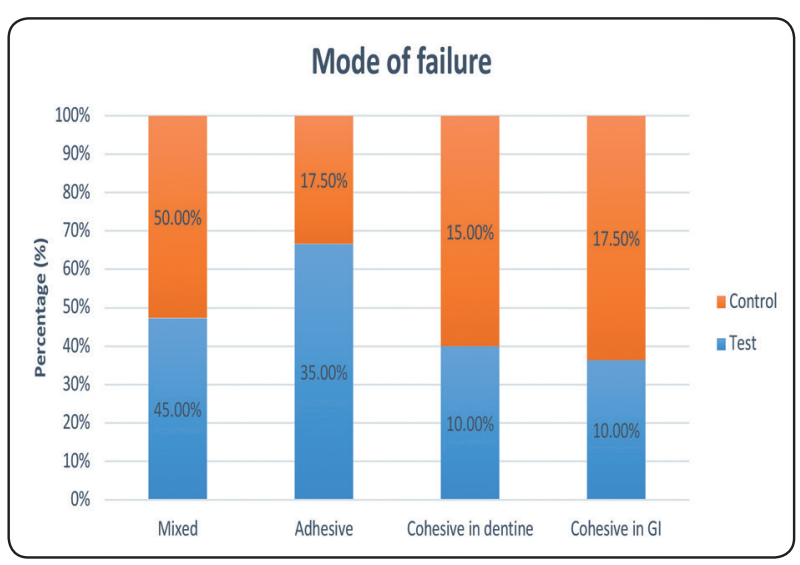

Fig. (10): Stacked bar chart showing percentage of mode of failure in both groups

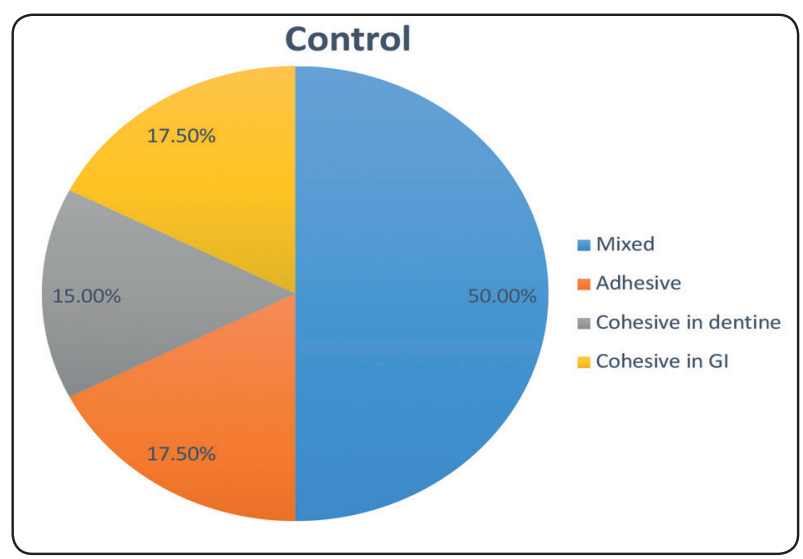

Fig. (11): Pie chart showing percentage of mode of failure in the control group

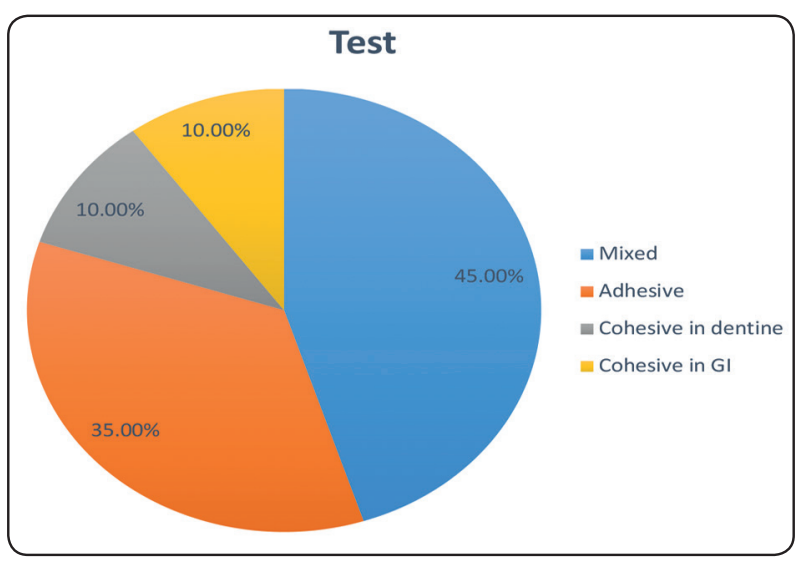

Fig. (12): Pie chart showing percentage of mode of failure in the test group

\section{DISCUSSION}

Caries prevalence and severity in primary teeth of children are high. Untreated tooth decay in the primary dentition is a common predictable phenomenon. The costly restorative treatment and the insufficient awareness with the oral health care, make it impracticable to control dental caries in children, especially in underdeveloped communities.

Evidence exists that fluoride varnish is considered a safe and effective agent for caries prevention in young children. SDF may be an alternative option for controlling tooth decay, especially at the cavitation level in preschool children. SDF may cause revolution in pediatric and community dentistry and may be a breakthrough dental agent for this century due to its safety, efficiency, feasibility and effectiveness in preventing and arresting dentin caries.

Although black staining is a known side effect of SDF, which can be minimized by certain modifications, particularly where access to traditional dental care is challenging whether due to financial or behavioral issues related to the children andlor their caregivers.

As noted earlier, the results from this study indicate no significant difference in bond strengths between the control group (no pretreatment with SDF) and test group (pretreatment with 38\% SDF). Based on the results of this study, the null hypothesis that SDF has no effect on the bond strength of glass ionomer to treated carious primary dentin is affirmed.

The main objective of this study was to investigate if pretreating carious primary dentin with SDF would adversely affect the bond strength between GIC and carious dentin. The clinical implication of such finding is that, if SDF is used to arrest and/ or prevent caries progression, the bond strength of GIC placed on the carious primary dentin of that tooth will not be affected. 
The present study used the microtensile bond test method. Both the control and the test specimen were obtained from the same tooth, thus, the tooth was its own control, which reduces some of the variability frequently observed in bond strength studies. The bonding surfaces in the present study are cariesaffected dentin judged using visual examination and surface hardness testing with a dental explorer.

Commercially available 38\% SDF solution (Riva Star, SDI) capsules that contains 44,800 ppm fluoride were used in the study. M Laboratory studies found that SDF has a significant antimicrobial effect against cariogenic biofilm ${ }^{(22,36)}$. Silver diamine fluoride treatment also possesses a remineralizing and rehardening effect on demineralized dentin ${ }^{(32,22)}$.

Previous studies found higher mineral contents in the dentin of carious lesions after treatment with SDF compared with normal dentin ${ }^{(32,22)}$.

A clinical study conducted by Sinha et al.,2011 and confirmed that SDF increased the level of calcium, phosphate, and fluoride ions in cariesaffected dentin, with the highest being fluoride ions $^{(33)}$.

It was postulated that SDF reacts with hydroxyapatite, forming fluoroapatite and insoluble silver phosphate $^{(32,22)}$.

In addition, SDF releases fluoride ions and helps in the deposition of silver phosphate to restore the mineral content, resulting in re-hardening of the soft tooth structure ${ }^{(37,36)}$.

After applying SDF, the prepared dentin surface for the bond test was blackened, which may occur by the precipitation of silver granules, resulting in the coagulation of exposed denatured collagen fibrils. This precipitate could result in a lower bond strength of a restorative material to SDF-modified dentin. Harnirattisai et al., 2007 demonstrated lower bond strengths of adhesive resins to amalgam-stained dentin (34).However, Knight et al., 2006 found that the application of silver fluoride/potassium iodide to etched dentin samples, followed by washing off the precipitate, did not affect the bond strength ${ }^{(30)}$.

The World Health Organization has recommended use of the atraumatic restorative technique (ART) for treatment of dental caries in children ${ }^{(35,36)}$. However, in infants and toddlers, ART is more complicated than SDF application.so the current study proposed for the use of SDF in these very young children to arrest caries. As the children age and become more cooperative, GIC restorations can be provided to this group. These offer the advantages of low cost and the possibility for application by dental personnel outside the traditional clinical setting, which may lead to an increase in dental service coverage. GIC is biocompatible, provides chemical adhesion to enamel and dentin in the presence of moisture, is resistant to microleakage with fluoride release, and rechargeable ${ }^{33,37)}$.

The basis of glass ionomer adhesion to teeth is believed to involve a chemical interaction with tooth structure via ion exchange. In addition to its micromechanical penetration into the tooth structure. ${ }^{(37)}$

An environment of fluoride hypersaturation created by SDF can lead to the formation of fluorapatite crystals in dentin, which are larger in size than hydroxyapatite crystals and, thus, have a closely packed structure with fewer voids and greater microhardness ${ }^{(32) .}$ From this morphological perspective, the bond strength in the SDF-treated group was expected to be lower because of fluoride hyper saturation. In our study, however, the bond strength SDF- treated carious dentin specimen was not different from the control group. It is possible that an SDF-modified dentin zone at the superficial level was partly removed when polished with 600grit silicon carbide paper, mitigating the effect of SDF on GIC bond strength.

The results of the present study show that SDF has no effect on the bond strength between glass ionomer and primary carious dentin. This finding agrees with results from a previous study, which revealed that SDF and potassium iodide had no 
effect on the bond strength of auto-cure GIC to non-carious permanent dentin ${ }^{(38)}$.Another study by Quock et al., 2012 found that SDF had no effect on the bond strength of composite resin to non-carious permanent dentin. ${ }^{(26)}$.

The bond strength of GIC to non-carious primary dentin in a previous study was higher than that in the present study ${ }^{(39)}$. This is normally expected, as the bond strength of GIC to caries-affected primary dentin would be less than that of non-carious dentin.

The modes of failure for the GIC/dentin bond were mostly mixed. A similar study testing the microtensile bond strength of GIC to normal primary dentin found that the majority of failures occurred cohesively in the GIC ${ }^{(39)}$.This may be related to the nature of the SDF-modified zone and the remaining carious dentin, which may be slightly more elastic in nature than the normal dentin. SDF has been used to arrest caries progression in the teeth of very young children in whom restorations are difficult to perform. When a child grows older and becomes more co-operative and accepting the dental procedures, restorations can be performed on the SDF-treated teeth using GIC. ${ }^{(22)}$.

\section{CONCLUSION}

Based on the present study's results, it can be concluded that Silver Diamine Fluoride with Potassium Iodide-treated carious primary dentin, does not adversely affect the bond strength of glass ionomer cement to carious primary dentin in vitro.

Although the results of the study indicates the bonding compatibility of glass ionomer cement to Silver Fluoride and Potassium Iodide-treated carious primary dentin, further investigation will be beneficial in determining the role of SDF in operative dentistry.

However, these laboratory findings should be complimented with longitudinal data from clinical studies investigating the failure of GIC restorations placed subsequent to SDF application.
When considering the clinical use of SDF, the present results suggest that, SDF and KI would not affect the bond strength between GIC and primary carious dentin. Clinical trials are necessary to determine if the efficacy of the bond observed in this study is maintained in the clinical setting.

\section{REFERENCES}

1. Selwitz RH, Ismail AI, Pitts NB. Dental-Caries-Seminar. pdf. Lancet. 2007;369(4):51-9.

2. Çolak H, Dülgergil Ç, Dalli M, Hamidi M. Early childhood caries update: A review of causes, diagnoses, and treatments. Vol. 4, Journal of Natural Science, Biology and Medicine. 2013. p. 29-38.

3. Featherstone JDB. The continuum of dental caries - Evidence for a dynamic disease process. In: Journal of Dental Research. 2004.

4. Fejerskov O, Nyvad B, Kidd EAM. Dental caries : the disease and its clinical management. $466 \mathrm{p}$.

5. Touger-Decker R, van Loveren C. Sugars and dental caries. Vol. 78, The American journal of clinical nutrition. 2003.

6. Ismail AI, Sohn W. A systematic review of clinical diagnostic criteria of early childhood caries. Vol. 59, Journal of Public Health Dentistry. Blackwell Publishing Inc.; 1999. p. 171-91.

7. Vos T, Abajobir AA, Abbafati C, Abbas KM, Abate KH, Abd-Allah F, et al. Global, regional, and national incidence, prevalence, and years lived with disability for 328 diseases and injuries for 195 countries, 1990-2016: A systematic analysis for the Global Burden of Disease Study 2016. Lancet. 2017 Sep 16;390(10100):1211-59.

8. Congiu G, Campus G, Lugliè PF. Early childhood caries (ECC) prevalence and background factors: A review. Oral Heal Prev Dent. 2014;12(1):71-6.

9. Horowitz HS. Research issues in early childhood caries. Community Dent Oral Epidemiol. 1998 Oct;26(S1):67-81.

10. Ismail AI, Lim S, Sohn W, Willem JM. Determinants of early childhood caries in low-income African American young children. Pediatr Dent. 2008;30(4):289-96.

11. Azizi Z. The prevalence of dental caries in primary dentition in 4- to 5-year-old preschool children in Northern Palestine. Int J Dent. 2014;2014. 
12. Elamin A, Garemo M, Gardner A. Dental caries and their association with socioeconomic characteristics, oral hygiene practices and eating habits among preschool children in Abu Dhabi, United Arab Emirates - the NOPLAS project. BMC Oral Health [Internet]. 2018 Dec 8 [cited 2019 Nov 2];18(1):104. Available from: https://bmcoralhealth. biomedcentral.com/articles/10.1186/s12903-018-0557-8

13. Abbass MMS, Mahmoud SA, El Moshy S, Rady D, Abubakr N, Radwan IA, et al. The prevalence of dental caries among egyptian children and adolescences and its association with age, socioeconomic status, dietary habits and other risk factors. A cross-sectional study [version 1; referees: 1 approved, 2 approved with reservations]. F1000Research. 2019;8.

14. BaniHani A, Deery C, Toumba J, Munyombwe T, Duggal M. The impact of dental caries and its treatment by conventional or biological approaches on the oral healthrelated quality of life of children and carers. Int J Paediatr Dent. 2018 Mar 1;28(2):266-76.

15. Feitosa S, Colares V, Pinkham J. The psychosocial effects of severe caries in 4-year-old children in Recife, Pernambuco, Brazil. Cad Saude Publica [Internet]. [cited 2019 Nov 18];21(5):1550-6. Available from: http://www.ncbi. nlm.nih.gov/pubmed/16158161

16. Schroth RJ, Levi J, Kliewer E, Friel J, Moffatt MEK. Association between iron status, iron deficiency anaemia, and severe early childhood caries: A case-control study. BMC Pediatr. 2013 Feb 7;13(1).

17. Niederman, R., Feres, M., Ogunbodede, E. Dentistry.. In: Debas, HT.Donkor, P.Gawande, A.Jamison, DT.Kruk, ME., Mock, CN., editors. Essential Surgery: Disease Control Priorities. The 2015 International Bank for Reconstruction and Development / The World Bank; Washington (DC): 2015. p. 173-95.

18. . Rosenblatt A, Stamford TC, Niederman R. Silver diamine fluoride: a caries "silver-fluoride bullet". J Dent Res. 2009; 88(2):116-25. [PubMed: 19278981]

19. Gao SS, Zhao IS, Hiraishi N, et al. Clinical Trials of Silver Diamine Fluoride in Arresting Caries among Children: A Systematic Review. JDR Clinical \& Translational Research. 2016

20. Mei, M.L.; Li, Q.L.; Chu, C.-H.; Lo, E.M.; Samaranayake, L.P. Antibacterial effects of silver diamine fluoride on multi-species cariogenic biofilm on caries. Ann. Clin. Microbiol. Antimicrob. 2013, 12, 4.
21. . Mei, M.L.; Chu, C.-H.; Low, K.H.; Che, C.M.; Lo, E.C.-M. Caries arresting effect of silver diamine fluoride on dentine carious lesion with S. mutans and L. acidophilus dualspecies cariogenic biofilm. Med. Oral Patol. Oral Cir. Bucal. 2013, 18, 824-831.

22. Chu, C.-H.; Mei, L.; Seneviratne, C.J.; Lo, E.C.-M. Effects of silver diamine fluoride on dentine carious lesions induced by Streptococcus mutans and Actinomyces naeslundii biofilms. Int. J. Paediatr. Dent. 2012, 22, 2-10.

23. . Mei, M.L.; Lo, E.C.-M.; Chu, C.-H. Clinical Use of Silver Diamine Fluoride in Dental Treatment, Compendium of continuing education in dentistry. Compend. Contin. Educ. Dent. 2016, 37, 93-98.

24. Chu, C.-H.; Lo, E.C.-M.; Lin, H. Effectiveness of silver diamine fluoride and sodium fluoride varnish in arresting dentin caries in Chinese pre-school children. J. Dent. Res. 2002, 81, 767-770.

25. Llodra, J.; Rodriguez, A.; Ferrer, B.; Menardia, V.; Ramos, T.; Morato, M. Efficacy of silver diamine fluoride for caries reduction in primary teeth and first permanent molars of schoolchildren: 36-month clinical trial. J. Dent. Res. 2005, 84, 721-724.

26. Quock, R.; Barros, J.; Yang, S.; Patel, S. Effect of silver diamine fluoride on microtensile bond strength to dentin. Oper. Dent. 2012, 37, 610-616.

27. Gotjamanos, T. Pulp response in primary teeth with deep residual caries treated with silver fluoride and glass ionomer cement ('atraumatic'technique). Aust. Dent. J. 1996, 41, 328-334.

28. Mei, M.L.; Zhao, I.S.; Ito, L.; Lo, E.C.; Chu, C.-H. Prevention of secondary caries by silver diamine fluoride. Int. Dent. J. 2015, 66, 71-77.

29. Knight, G.; Mclntyre, J.; Craig, G. Ion uptake into demineralized dentine from glass ionomer cement following pretreatment with silver fluoride and potassium iodide. Aust. Dent. J. 2006, 51, 237-241.

30. Knight, G.; Mclntyre, J. The effect of silver fluoride and potassium iodide on the bond strength of auto cure glass ionomer cement to dentine. Aust. Dent. J. 2006, 51, 42-45.

31. Zafar, M.S.; Ahmed, N. Therapeutic roles of fluoride released from restorative dental materials. Fluoride 2015, 48, 184-194.

32. Gupta A, Sinha N, Logani A, Shah N. An ex vivo studyto evaluate the remineralizing and antimicrobial efficacyof 
silver diamine fluoride and glass ionomer cement type VII for their proposed use as indirect pulp capping materials: part I. J Conserv Dent 2011;14(2):113-6.

33. Sinha N, Gupta A, Logani A, Shah N. Remineralizing efficacy of silver diamine fluoride and glass ionomer type VII for their proposed use as indirect pulp capping materials: part II (A clinical study). J Conserv Dent 2011; 14(3): 233-6.

34. Harnirattisai C, Senawongse P, Tagami J. Microtensile bond strengths of two adhesive resins to discolored dentin after amalgam removal. J Dent Res 2007; 86(3):232-6.

35. Frencken J, Pilot T, Songpaisan Y, Phantumvanit P. Atraumatic restorative treatment (ART): rationale, technique, and development. J Public Health Dent 1996; 56(3 Spec No):135-40, discussion 161-3.
36. Frencken J. Manual for the atraumatic restorative treatment approach to control dental caries. 3rd ed. Groningen, The Netherlands: World Health Organization Collaborating Centre for Oral Health Services Research; 1997: 2, 16.

37. Saito S, Tosaki S, Hirota K. Characteristics of glass ionomer cements. In: Davidson CL, Major IA, eds. Advances in Glass Ionomer Cements. Batavia, Il: Quintessence Publishing Company; 1999:15-50.

38. Wu DI, Velamakanni S, Denisson J, et al. Effect of silver diamine fluoride (SDF) application on microtensile bonding strength of dentin in primary teeth. Pediatr Dent 2016; 38(2):148-53.

39. Burrow MF, Nopnakeepong U, Phrukkanon S. A comparison of microtensile bond strengths of several dentin bonding systems to primary and permanent dentin. Dent Mater 2002; 18(3):239-45. 